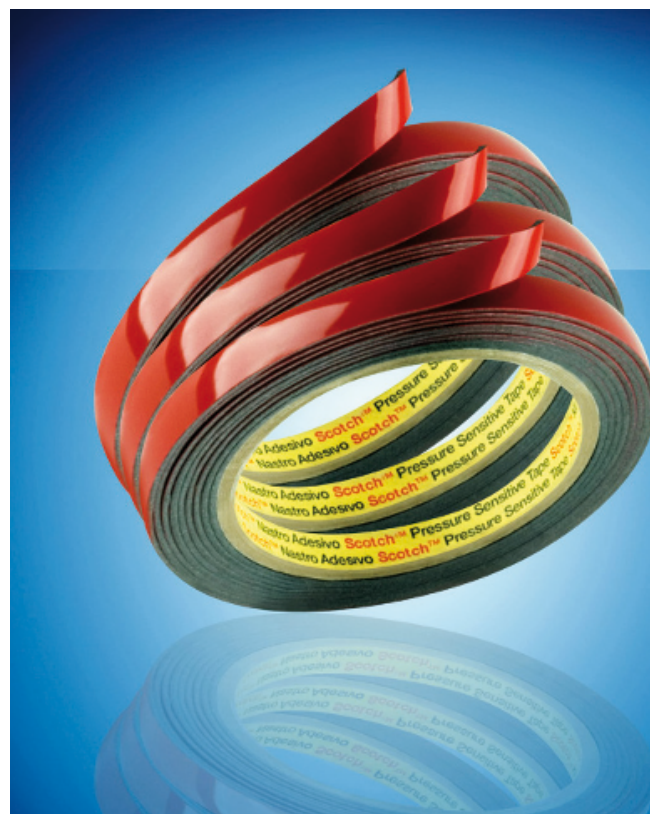

\title{
Reliable adhesion on modern coating systems
}

$3^{\mathrm{M}}$ has added a new $1.5 \mathrm{~mm}$ thick product to its EX range of adhesive tapes 3 used for fastening plastic trim parts to vehicles. As a result, acrylic tapes are now available in thicknesses of $0.8 \mathrm{~mm}, 1.1 \mathrm{~mm}$ and $1.5 \mathrm{~mm}$ for this application.

Like the other two products in the range, the new adhesive tape offers excellent adhesion on modern automotive paint systems on the side covered by a protective liner. The open side of the tape has been designed to bond with the plastic trim components. It is ideal for joining medium-surface-energy substrates, such as ABS plastics, and a wide variety of other surfaces, including paint systems. In addition to offering excellent adhesion to a range of different substrates, the product can also compensate for tolerance variations because of its dark grey foam core made from acrylic adhesive. The thicker the tape, the larger the tolerances that it can accommodate. Because of its inner strength, the acrylic foam tape also has excellent long-term stability. It is suitable for both manual and automated application processes.

For more information: $3 \mathrm{M}$ Deutschland $\mathrm{GmbH}$,

Tel. +49 (0)2131 14 2216, jklueppelberg@mmm.com

\section{New glue gun processes a wide variety of hot-melts}

$\triangle$ mechanical hot glue gun, which can Abe used to process hot-melt adhesives in almost all their available forms, including granules, pillows and sticks, without compressed air, was presented for the first time at the Drupa trade fair.

Hot-melt adhesives are used in a wide range of industries to bond many different materials, for example in prepress for producing samples, in the automotive industry for bonding interior components and in the packaging industry for fastening cardboard boxes. The benefits of these adhesives include their versatility, lack of solvents, affordable price and short setting time. This is particularly important in the manufacture of samples where different materials have to be bonded quickly

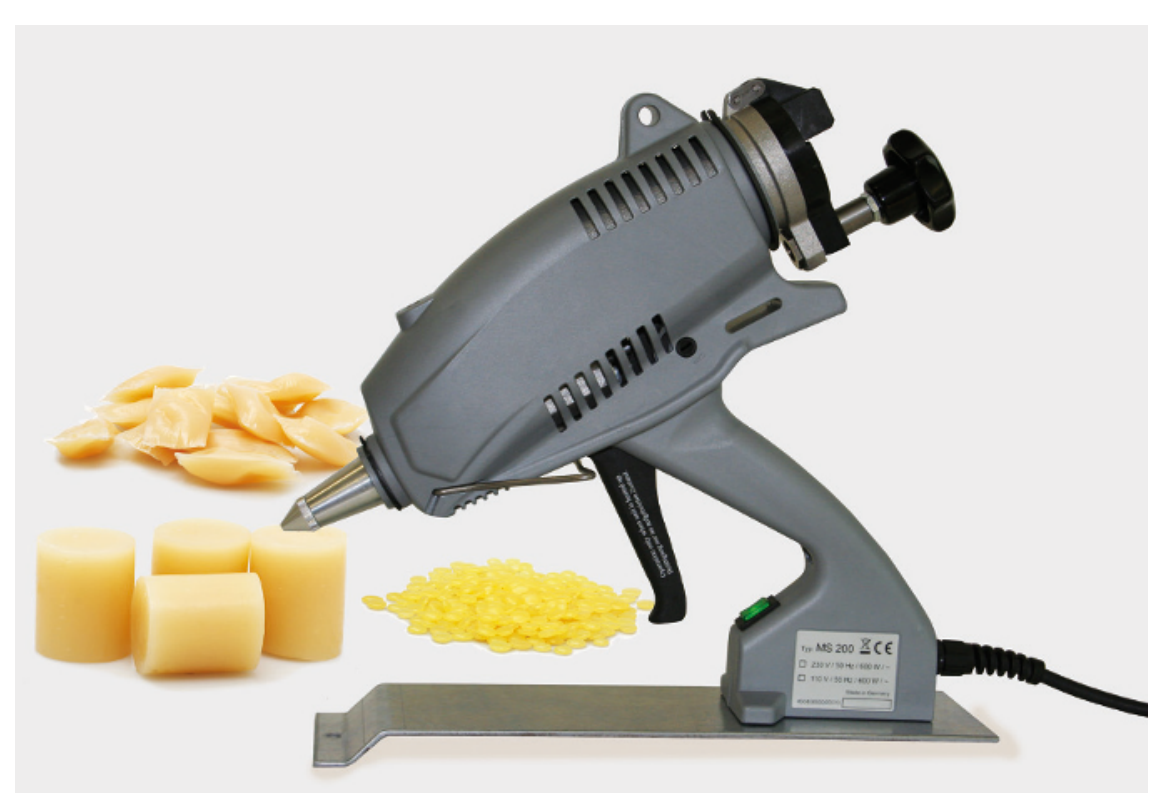
and cleanly.

In an industrial context, hot-melts are generally applied with tank systems, which have the advantage that they can process all the common forms of the adhesive. However, tank systems have a high purchase cost and are also expensive to maintain. In contrast, for DIY purposes hot-melts can be applied with simple stick devices which are low in cost and easy to use. However, only a very limited range of hot-melt adhesives is available in stick form. For this reason, Reka Klebetechnik has developed the new MS 200 mechanical hot glue gun. It is a cost-effective and flexible solution for applying almost all the industrial forms of hot-melt ad-

hesive, such as granules, pillows and 43 mm sticks, without compressed air. The ergonomic design and the large tank containing the melted adhesive enable the user to apply the adhesive without become tired and without the need for interruptions. A number of useful accessories also make the work easier, even in complicated applications. The comprehensive range of nozzles available meets almost every requirement.

For more information: Reka-Klebetechnik GmbH \& Co. KG, D-76344 Eggenstein, Germany, Tel.: +49 (0)721970 780, sale@reka-klebetechnik.de 